\title{
Patients with Head-and-Neck Cancer: Dysphagia and Affective Symptoms
}

\author{
Iris Krebbers ${ }^{\mathrm{a}}$, Sorina R. Simon ${ }^{\mathrm{a}} \quad$ Walmari Pilz $^{\mathrm{a}} \mathrm{b}$ Bernd Kremer ${ }^{\mathrm{a}}$ \\ Bjorn Winkens ${ }^{c, d}$ Laura W.J. Baijens ${ }^{a, b}$
}

a Department of Otorhinolaryngology, Head and Neck Surgery, Maastricht University Medical Center, Maastricht, The Netherlands; 'b School for Oncology and Developmental Biology, GROW, Maastricht University Medical Center, Maastricht, The Netherlands; ' Department of Methodology and Statistics, Maastricht University, Maastricht, The Netherlands; ${ }^{\mathrm{d} C a r e}$ and Public Health Research Institute, CAPHRI, Maastricht University Medical Center, Maastricht, The Netherlands

\section{Keywords \\ Dysphagia - Aspiration · Head and neck cancer · Hospital Anxiety and Depression Scale · Fiberoptic endoscopic evaluation of swallowing}

\begin{abstract}
Objective: Affective symptoms are common in patients with head-and-neck cancer. This study determined the association between the presence of aspiration and symptoms of anxiety and depression, as well as patient characteristics in patients with head-and-neck cancer and dysphagia. Methods: Eighty-four patients with head-and-neck cancer and dysphagia completed the Hospital Anxiety and Depression Scale and underwent a standardized fiberoptic endoscopic evaluation of swallowing. Linear regression analysis was performed to explore the associations. Results: Fifty-two (61.9\%) patients presented clinically relevant symptoms of anxiety or depression. Forty-eight (57.1\%) patients presented with aspiration during fiberoptic endoscopic evaluation of swallowing. A significant negative association was found between the presence of aspiration and affective (anxiety and depression) symptoms $(p=0.04)$. Male patients presented significantly lower symptom scores of anxiety compared to females
\end{abstract}

( $p=0.04)$. Conclusions: Clinically relevant affective symptoms were present in more than half of all patients with headand-neck cancer and dysphagia. Surprisingly, a significant negative association was found between the presence of aspiration and these affective symptoms. Gender was also significantly associated with affective symptoms. These results suggest that there is a need for further investigation into the impact of psychological distress on patients with head-andneck cancer and dysphagia.

(C) 2020 The Author(s)

Published by S. Karger AG, Basel

\section{Introduction}

Oropharyngeal dysphagia (OD) is common in patients with head-and-neck cancer (HNC). Swallowing function may be affected by oncological treatment such as surgery with or without (neo)adjuvant (chemo)radiotherapy or definitive (chemo)radiotherapy [1]. The main OD complaints are difficulty in chewing, regurgitation, coughing, odynophagia, and choking while eating [2]. OD severity can vary among patients. Aspiration seems to be the most critical marker of OD since it might increase the risk of severe complications such as aspiration pneumonia (as-
(C) 2020 The Author(s)

Published by S. Karger AG, Basel

This article is licensed under the Creative Commons AttributionNonCommercial-NoDerivatives 4.0 International License (CC BYNC-ND) (http://www.karger.com/Services/OpenAccessLicense) Usage and distribution for commercial purposes as well as any distribution of modified material requires written permission.
Iris Krebbers

Department of Otorhinolaryngology, Head and Neck Surgery

Maastricht University Medical Center

PO Box 5800, NL-6202 AZ Maastricht (The Netherlands)

iris.krebbers@mumc.nl 
sociated with a mortality range from 20 to $65 \%$ ) and malnutrition due to restrictive dietary adaptations made by the patient $[3,4]$.

Besides these physical consequences, OD is also accompanied by anxiety, reduced self-esteem, and social isolation [5-8]. The recognition and treatment of the psychosocial burden in patients with HNC is important as distress may interfere with their ability to cope with the disease and its treatment [9]. In diverse oncological healthcare settings (pre-, during, and post-treatment), the Hospital Anxiety and Depression Scale (HADS) is frequently used to screen for clinically relevant affective symptoms [10-13]. Anxiety and depression are associated with decreased health-related quality of life (HRQoL), non-adherence to rehabilitation programs, and increased use of healthcare services $[8,9,14]$. The impact of OD on symptoms of anxiety, depression, and HRQoL has already been studied in patients with HNC; the severity of OD has been correlated with a compromised HRQoL and clinically relevant affective symptoms $[15,16]$. However, the presence of $\mathrm{OD}$ has a wide-ranging impact on patients with HNC. A significant relationship is expected between aspiration and clinically relevant levels of anxiety and depressive symptoms in patients with HNC. There is a need for further investigation into the severity of OD and its impact on psychological distress to provide appropriate integrated care in this vulnerable population. A more complete understanding of the prevalence of affective symptoms, such as symptoms of anxiety and depression, in patients with HNC and OD can support allied health professionals in developing sustainable custom-made multidisciplinary OD support.

The aim of this study was to determine the association between the presence of aspiration and symptoms of anxiety and depression, as well as demographic characteristics (age, gender), level of the Functional Oral Intake Scale (FOIS), type of HNC treatment, and tumor location in patients with HNC and OD.

\section{Materials and Methods}

\section{Study Population}

For this cross-sectional cohort study, patients with HNC and OD were recruited from the outpatient clinic of the Department of Otorhinolaryngology at a tertiary university referral hospital between November 2011 and February 2016. Patients were referred by their speech and language pathologist who had identified symptoms of OD. Individuals were enrolled in the study if they had completed the HNC treatment (surgery, radiotherapy, chemoradiotherapy, or combinations thereof - multimodality treatment) at least 6 months before recruitment and their disease was in total remission (i.e., they were disease free). Several exclusion criteria were applied: severe odynophagia (unable to swallow); radiation mucositis; more than 1 primary tumor in the head and neck region; osteoradionecrosis of the maxilla or mandible; presenting with a concurrent neurological disease (stroke, Parkinson disease, etc.); scoring below 23 on the Mini-Mental State Examination (MMSE) [17]; being older than 85 years; having undergone a total laryngectomy; and being illiterate or blind. All primary tumors were classified according to the TNM classification, 7th edition [18]. The study protocol was approved as non-WMO (Wet Medisch-Wetenschappelijk Onderzoek) research by the medical ethics committee in compliance with the Medical Research Involving Human Subjects Act (WMO) [19].

In total, 84 patients with $\mathrm{HNC}$ and complaints of OD were enrolled in the study. The mean (SD) age of the study population was 65.8 (10) years. The median (IQR) FOIS score was 5 (4-6). Ten (11.9\%) patients were using psychotropic medication (atypical antipsychotics or selective serotonin reuptake inhibitors) at the time of the fiberoptic endoscopic evaluation of swallowing (FEES) examination. An overview of the patient characteristics is given in Table 1.

\section{Examination Protocol}

All patients underwent the standardized examination protocol used in daily clinical practice at the outpatient clinic for OD, thereby providing prospectively collected data. This protocol comprises a structured interview, the HADS questionnaire, the MMSE, a standardized otorhinolaryngological examination, a standardized FEES examination, and the FOIS. The FOIS is a scale that indicates the clinically relevant level of dietary intake. Its scores range from 1 (nothing by mouth/ tube feeding only) to 7 (total oral diet with no restrictions) [20]. At levels 1-3 there is tube feeding dependency and levels 4-7 indicate a total oral diet. The HADS questionnaire is a validated screening tool for clinically relevant symptoms of anxiety or depression, also named affective symptoms [21]. Of its 14 items, 7 are related to anxiety symptoms (HADS-A) and 7 to depressive symptoms (HADS-D). Each item is scored from 0 to 3, depending on the severity of the symptoms, where 0 indicates their absence and 3 almost continuous presence of symptoms. The sum of the scores ranges from 0 to 21 for either the HADS-A or HADSD subscale, and a maximum of 42 points for the HADS total score (HADS-T). A score of 8 or more on each subscale indicates the presence of clinically relevant symptoms of anxiety or depression $[21,22]$. A translated and validated Dutch version of the HADS questionnaire was used in this study [23]. All HADS and FEES examinations were performed at least 6 months after completion of the HNC treatment: median (IQR) 42 months (7.5-122).

The FEES examinations were carried out by an experienced laryngologist together with a speech and language pathologist. During the examination all patients performed 3 cued swallows of $10 \mathrm{~mL}$ of thin liquid (water) followed by 3 cued swallows of $10 \mathrm{~mL}$ of thick liquid (applesauce; One 2 fruit $^{\circledR}$ ). All liquids were dyed with $5 \%$ methylene blue $(10 \mathrm{mg} / \mathrm{mL})$. The viscosity of the liquid bolus consistencies was measured at $25^{\circ} \mathrm{C}$ and $50 \mathrm{~s}^{-1}$ of shear rate resulting in $1 \mathrm{mPa} \bullet \mathrm{s}$ for thin liquid and $1,200 \mathrm{mPa} \bullet \mathrm{s}$ for thick liquid. During the flow test, the thick liquid met the criteria for "moderately thick" according to the International Dysphagia Diet Standardisation Initiative (IDDSI) [24]. A flexible fiberoptic endoscope, Pentax FNL-10RP3 (Pentax Canada Inc., Mississauga, ON, Canada), was used during the FEES examination. The tip of the 
Table 1. Patient characteristics (total number of patients $=84$ )

\begin{tabular}{|c|c|}
\hline Patient characteristics & $n(\%)$ \\
\hline \multicolumn{2}{|l|}{ Gender } \\
\hline Male & $58(69)$ \\
\hline Female & $26(31)$ \\
\hline \multicolumn{2}{|l|}{ T classification } \\
\hline $\mathrm{T} 1$ & $16(21.9)$ \\
\hline $\mathrm{T} 2$ & $19(26.0)$ \\
\hline T3 & $16(21.9)$ \\
\hline $\mathrm{T} 4$ & $20(27.4)$ \\
\hline Tis & $1(1.4)$ \\
\hline Tx (unknown primary) & $1(1.4)$ \\
\hline Missing & 11 \\
\hline \multicolumn{2}{|l|}{$\mathrm{N}$ classification } \\
\hline N0 & $41(57.9)$ \\
\hline N1 & $7(9.8)$ \\
\hline N2 & $22(30.9)$ \\
\hline N3 & $1(1.4)$ \\
\hline Missing & 13 \\
\hline \multicolumn{2}{|l|}{ M classification } \\
\hline M0 & $84(100)$ \\
\hline \multicolumn{2}{|l|}{ Therapy } \\
\hline Definitive radiotherapy (single modality) & $29(34.5)$ \\
\hline Definitive chemoradiotherapy & $17(20.2)$ \\
\hline Surgery (single modality) & $8(9.5)$ \\
\hline Surgery and adjuvant (chemo)radiotherapy & $30(35.7)$ \\
\hline \multicolumn{2}{|l|}{ Tumor location } \\
\hline Pharynx & $33(39.3)$ \\
\hline Larynx & $30(35.7)$ \\
\hline Oral cavity & $13(15.5)$ \\
\hline Other location ${ }^{\mathrm{a}}$ & $8(9.5)$ \\
\hline \multicolumn{2}{|l|}{ Tumor histopathology } \\
\hline Squamous cell carcinoma & $64(87.7)$ \\
\hline Adenocarcinoma & $2(2.7)$ \\
\hline Verrucous carcinoma & $1(1.4)$ \\
\hline Other histopathology & $6(8.2)$ \\
\hline Missing & 11 \\
\hline \multicolumn{2}{|l|}{ FOIS $^{\mathrm{b}}$} \\
\hline Level 1 & $9(10.7)$ \\
\hline Level 2 & $6(7.1)$ \\
\hline Level 3 & $1(1.2)$ \\
\hline Level 4 & $6(7.1)$ \\
\hline Level 5 & $34(40.5)$ \\
\hline Level 6 & $19(22.6)$ \\
\hline Level 7 & $9(10.7)$ \\
\hline \multicolumn{2}{|l|}{ Aspiration } \\
\hline Yes & $48(57.1)$ \\
\hline No & $36(42.9)$ \\
\hline \multicolumn{2}{|l|}{ Psychotropic medication } \\
\hline Yes & $10(11.9)$ \\
\hline No & $74(88.1)$ \\
\hline
\end{tabular}

a Nasal (sinus) cavity, salivary glands. ${ }^{\mathrm{b}}$ FOIS, Functional Oral Intake Scale. endoscope was in "high position," just above the epiglottis, where the scope could not interfere with closure of the laryngeal vestibule [25]. The FEES videos were obtained with the Xion SD camera, Xion EndoSTROBE camera control unit (PAL $25 \mathrm{fps}$ ), and Matrix DS data station with DIVAS software (Xion Medical, Berlin, Germany) and recorded on a DVD. No topical anesthetic or nasal vasoconstrictor was used during the procedure. For each FEES swallow the visuoperceptual ordinal variable "aspiration" was scored dichotomously (present vs. absent) by 2 observers who had followed the training program described in previous studies [26, 27]. Aspiration was defined as entry of the bolus into the larynx below the level of the vocal folds including bolus at the level of the vocal commissures during the swallow as described in a previous observer agreement study $[28,29]$. The observers were blinded to the patients' identity, medical history, HADS scores, and to each other's rating scores (independent rating). All swallows were evaluated twice by observer 1 (blinded, in randomized order and with a time lag of 2 weeks) to assess intraobserver agreement. Half of all FEES trials were scored by a second independent observer to determine interobserver agreement. Each observer was asked to limit the evaluation period to a maximum of $2 \mathrm{~h}$ in order to maintain optimal attention and reduce fatigue-related bias.

\section{Statistical Analysis}

Descriptive statistics were presented as numbers and percentages for categorical variables and as the mean (SD) or median (IQR) for numerical variables. The statistical analysis was performed on the presence of aspiration independent of the bolus consistency. For the visuoperceptual ordinal FEES variable aspiration, both inter- and intraobserver agreement were calculated using the linear weighted kappa coefficient [30]. Interobserver agreement levels of the FEES outcome variable aspiration were substantial $(\kappa=0.76)$. Intraobserver agreement levels for observer 1 and observer 2 were $\kappa=0.81$ and $\kappa=0.71$, respectively.

The possible association between the presence of aspiration and symptoms of anxiety and depression, as well as demographic factors (age, gender), level of the FOIS, type of HNC treatment, and tumor location was assessed using linear regression analyses, where aspiration (yes/no), gender (male/female), age (in years), type of oncological treatment (surgery with or without (neo)adjuvant (chemo)radiotherapy or definitive (chemo)radiotherapy, etc.), tumor location (pharynx, larynx, oral cavity, or other location), and FOIS (level 1-3/level 4-7) were included in the model. Adjusted estimated associations (regression coefficients) were reported together with their $95 \% \mathrm{CI}$ and $p$ values. Two-sided $p$ values $\leq 0.05$ were considered statistically significant. Missing data were not imputed. Variables included in the regression analyses did not contain any missing data. All statistical analyses were performed using IBM SPSS Statistics for Windows, version 24.0 (IBM, Armonk, NY, USA).

\section{Results}

\section{HADS Questionnaire}

All patients completed the HADS questionnaire. Fiftytwo (61.9\%) patients scored 8 or more points on the HADS subscales, indicating the presence of clinically rel- 
Table 2. Association between patient characteristics and HADS-A scores

\begin{tabular}{|c|c|c|c|}
\hline Outcome HADS-A & $\begin{array}{l}\text { Observed mean } \\
\text { HADS-A (SD) }\end{array}$ & $\begin{array}{l}\text { Estimated association }^{\mathrm{a}} \\
(95 \% \mathrm{CI})\end{array}$ & $p$ value \\
\hline Aspiration & & & 0.05 \\
\hline Yes $(n=48)$ & $7.3(4.5)$ & $-2.0(-4.1$ to 0.0$)$ & \\
\hline No $(n=36)$ & $9.1(4.3)$ & Ref & \\
\hline Age, years & & $0.1(-0.02$ to 0.2$)$ & 0.10 \\
\hline Gender & & & 0.01 \\
\hline Male $(n=58)$ & $7.2(4.2)$ & $-2.8(-5.0$ to -0.6$)$ & \\
\hline Female $(n=26)$ & $10(4.4)$ & Ref & \\
\hline Therapy & & & 0.90 \\
\hline Definitive radiotherapy $(n=29)$ & $8.2(4.1)$ & Ref & \\
\hline Definitive chemoradiotherapy $(n=17)$ & $7.6(3.5)$ & $0.0(-2.9$ to 2.9$)$ & \\
\hline Surgery (single modality) $(n=8)$ & $9.6(4.8)$ & $0.8(-3.8$ to 5.4$)$ & \\
\hline Surgery and (chemo)radiotherapy $(n=30)$ & $7.8(5.3)$ & $-0.5(-3.3$ to 2.2$)$ & \\
\hline Tumor location & & & 0.94 \\
\hline Pharynx $(n=33)$ & $7.7(4.2)$ & $0.6(-1.9$ to 3.1$)$ & \\
\hline Larynx $(n=30)$ & $7.9(4.5)$ & Ref & \\
\hline Oral cavity $(n=13)$ & $9.1(5.0)$ & $1.0(-2.9$ to 4.9$)$ & \\
\hline Other locations $\mathrm{s}^{\mathrm{b}}(n=8)$ & $8.8(5.2)$ & $0.2(-3.8$ to 4.1$)$ & \\
\hline FOIS & & & 0.64 \\
\hline $1-3(n=16)$ & $8.1(5.0)$ & Ref & \\
\hline $4-7(n=68)$ & $8.1(4.4)$ & $-0.6(-3.2$ to 2.0$)$ & \\
\hline
\end{tabular}

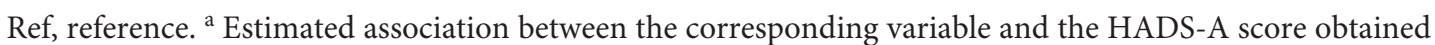
using multiple linear regression analyses after adjustment for the other variables mentioned in this table. ${ }^{\text {b Nasal }}$ (sinus) cavity, salivary glands.

evant symptoms of anxiety or depression. Clinically relevant symptoms of anxiety were present in 39 (46.4\%) patients and clinically relevant symptoms of depression were present in $46(54.7 \%)$ patients. The median (IQR) HADS-A and HADS-D scores were 7 (5-11) and 8 (4$10)$. No floor or ceiling effects were found, as few patients $(<2.0 \%)$ had the highest or the lowest score on the questionnaire. The median (IQR) HADS-A and HADS-D scores of patients using psychotropic drugs were 13 (11$15)$ and 11 (9-16), respectively, and 7 (4-11) and 7 (4-9) for patients who did not use psychotropic drugs.

\section{Association between Patient Characteristics and HADS Scores}

Forty-eight (57.1\%) patients presented with aspiration of at least one bolus consistency during the FEES examination. The adjusted associations between aspiration and patient characteristics, such as gender, age, level of the FOIS, type of HNC treatment, and tumor location versus the HADS scores are shown in Table 2 (HADS-A), Table 3 (HADS-D), and Table 4 (HADS-T). Few statistically significant associations were found. The associations between aspiration and HADS-A, HADS-D, and HADS-T scores were statistically significant $(p=0.05, p=0.04, p=$ 0.04 ). Compared to dysphagic patients who did not aspirate, dysphagic patients presenting with aspiration scored on average 2.0, 2.2, and 4.2 points lower on the HADS-A, HADS-D, and HADS-T scale, representing lower symptom scores for anxiety and depression. Significant associations between gender and HADS-A and HADS-T scores were also found ( $p=0.01,0.04)$. Male patients scored on average 2.8 points lower on the HADS-A scale compared to females, indicating that the men had significantly lower symptom scores on anxiety than the women. Other patient characteristics such as age, level of the FOIS, type of HNC treatment, and tumor location were not significantly associated with HADS-A, HADS-D, or HADS-T scores.

\section{Discussion}

This cross-sectional cohort study described the association between the presence of aspiration and symptoms of anxiety and depression, as well as demographic factors (age, gender), level of the FOIS, type of HNC treatment, and tu- 
Table 3. Association between patient characteristics and HADS-D scores

\begin{tabular}{|c|c|c|c|}
\hline Outcome HADS-D & $\begin{array}{l}\text { Observed mean } \\
\text { HADS-D (SD) }\end{array}$ & $\begin{array}{l}\text { Estimated association }{ }^{\mathrm{a}} \\
(95 \% \mathrm{CI})\end{array}$ & $p$ value \\
\hline Aspiration & & $-2.2(-4.3$ to -0.1$)$ & 0.04 \\
\hline Yes $(n=48)$ & $6.7(4.6)$ & Ref & \\
\hline No $(n=36)$ & $8.8(3.8)$ & & \\
\hline Age, years & & $0.1(-0.1$ to 0.2$)$ & 0.37 \\
\hline Gender & & & 0.18 \\
\hline Male $(n=58)$ & $7.1(4.2)$ & $-1.5(-3.7$ to 0.7$)$ & \\
\hline Female $(n=26)$ & $8.6(4.8)$ & Ref & \\
\hline Therapy & & & 0.70 \\
\hline Definitive radiotherapy $(n=29)$ & $6.9(4.5)$ & Ref & \\
\hline Definitive chemoradiotherapy $(n=17)$ & $7.9(4.3)$ & $1.0(-2.0$ to 3.9$)$ & \\
\hline Surgery (single modality) $(n=8)$ & $9.3(3.8)$ & $1.2(-3.5$ to 5.8$)$ & \\
\hline Surgery and (chemo)radiotherapy $(n=30)$ & $7.6(4.6)$ & $0.3(-2.5$ to 3.1$)$ & \\
\hline Tumor location & & & 0.90 \\
\hline Pharynx $(n=33)$ & $7.6(4.7)$ & $0.9(-1.7$ to 3.5$)$ & \\
\hline Larynx $(n=30)$ & $7.0(4.4)$ & Ref & \\
\hline Oral cavity $(n=13)$ & $9.0(4.2)$ & $1.4(-2.5$ to 5.4$)$ & \\
\hline Other locations $^{\mathrm{b}}(n=8)$ & $7.5(3.6)$ & $-0.7(-4.8$ to 3.3$)$ & \\
\hline FOIS & & & 0.89 \\
\hline $1-3(n=16)$ & $7.1(4.7)$ & Ref & \\
\hline $4-7(n=68)$ & $7.7(4.4)$ & $0.2(-2.5$ to 2.8$)$ & \\
\hline
\end{tabular}

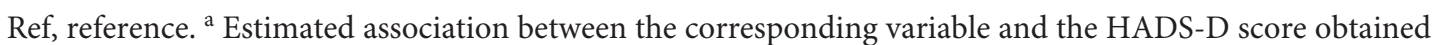
using multiple linear regression analyses after adjustment for the other variables mentioned in this table. ${ }^{b}$ Nasal (sinus) cavity, salivary glands.

mor location. More than half of all participants showed clinically relevant affective symptoms (HADS A/D $>8$ ). The results of this study showed that the presence of aspiration was accompanied by significantly lower scores on affective symptoms. Furthermore, male patients presented significantly lower symptom scores for anxiety compared to female patients with HNC. On the other hand, age, level of the FOIS, type of HNC treatment, and tumor location were not significantly associated with the HADS scores.

A diagnosis of cancer is accompanied by a high level of distress, which can manifest itself in symptoms of anxiety and depression [31]. These may increase due to disease progression, physical symptoms caused by the disease such as fatigue, visibility, and impairment of basic functions such as eating and speaking [32]. The recognition and treatment of clinically relevant anxiety and depression symptoms in patients with HNC and OD is important as these symptoms may inhibit their capacity for coping with the disease and its treatment. The HADS questionnaire is one of the most frequently used tools to measure symptoms of anxiety and depression in oncologic patients. Zigmond and Snaith [21] defined a HADS score of $\geq 8$ as the cut-off point for clinically relevant
HADS-A and HADS-D scores. In the current study, this cut-off value of 8 with only HADS scores $\geq 8$ was not applied in the linear regression analysis in an effort to obtain the highest possible statistical power. The whole range of scores of the HADS scales was used in the statistical analysis to explore the association between the entire severity range of affective symptoms and aspiration in this HNC population. A previous study determined a minimal clinically important difference of 1.7 for the HADS subscales in patients with cardiovascular disease, representing the smallest change in a HADS outcome that an individual patient would identify as important [33]. In the present study, the average score differences on the HADS scales between non-aspirating patients with OD versus aspirating patients were all higher than 1.7 points. So, the counter-intuitive negative association between aspiration and affective symptoms seems to be clinically meaningful in this patient group.

Aspiration during the FEES examination occurred in more than half of the study population. This finding corresponds with the frequencies of aspiration reported in previous studies (36-94\%) [34, 35]. In the present study, the finding of aspiration during FEES was accompanied 
Table 4. Association between patient characteristics and HADS-T scores

\begin{tabular}{|c|c|c|c|}
\hline Outcome HADS-T & $\begin{array}{l}\text { Observed mean } \\
\text { HADS-T (SD) }\end{array}$ & $\begin{array}{l}\text { Estimated } \\
\text { association }^{\text {a }}(95 \% \text { CI })\end{array}$ & $p$ value \\
\hline Aspiration & & & 0.04 \\
\hline Yes $(n=48)$ & $14(8.7)$ & $-4.2(-8.1$ to -0.3$)$ & \\
\hline No $(n=36)$ & $17.8(7.6)$ & Ref & \\
\hline Age, years & & $0.1(-0.1$ to 0.3$)$ & 0.18 \\
\hline Gender & & & 0.04 \\
\hline Male $(n=58)$ & $14.3(8.1)$ & $-4.3(-8.4$ to -0.2$)$ & \\
\hline Female $(n=26)$ & $18.7(8.6)$ & Ref & \\
\hline Therapy & & & 0.83 \\
\hline Definitive radiotherapy $(n=29)$ & $15.1(8.3)$ & Ref & \\
\hline Definitive chemoradiotherapy $(n=17)$ & $15.5(7.2)$ & $1.0(-4.6$ to 6.5$)$ & \\
\hline Surgery (single modality; $n=8$ ) & $18.9(8.3)$ & $2.0(-6.8$ to 10.8$)$ & \\
\hline Surgery and (chemo)radiotherapy $(n=30)$ & $15.4(9.4)$ & $-0.2(-5.4$ to 5.1$)$ & \\
\hline Tumor location & & & 0.92 \\
\hline Pharynx $(n=33)$ & $15.2(8.5)$ & $1.5(-3.4$ to 6.4$)$ & \\
\hline Larynx $(n=30)$ & $14.9(8.4)$ & Ref & \\
\hline Oral cavity $(n=13)$ & $18.1(8.9)$ & $2.4(-5.0$ to 9.8$)$ & \\
\hline Other locations $\mathrm{s}^{\mathrm{b}}(n=8)$ & $16.3(8.4)$ & $-0.6(-8.2$ to 7.0$)$ & \\
\hline FOIS & & & 0.87 \\
\hline $1-3(n=16)$ & $15.2(9.4)$ & Ref & \\
\hline $4-7(n=68)$ & $15.8(8.2)$ & $-0.4(-5.4$ to 4.6$)$ & \\
\hline
\end{tabular}

Ref, reference. ${ }^{\text {a }}$ Estimated association between the corresponding variable and the HADS-T score obtained using multiple linear regression analyses after adjustment for the other variables mentioned in this table. ${ }^{\text {b Nasal }}$ (sinus) cavity, salivary glands.

by significantly lower scores on self-reported symptoms of anxiety and depression compared to the HADS scores of non-aspirating patients with $\mathrm{HNC}$ and OD. A possible explanation for this counter-intuitive finding might lie in a decreased laryngeal sensitivity due to the oncological treatment, which reduces the patient's perception of swallowing impairment $[36,37]$. Moreover, a previous study on the perspective of patients with OD and of caregivers reported that depression is more likely to occur when the impairment has a higher impact on the patient's well-being and not only on the swallowing function itself, for instance, when patients are not able to eat their favorite food, or when they feel embarrassed and avoid eating with family or friends [38]. Another possible explanation may be related to the time interval between the end of the HNC treatment and the period of data collection. All HADS and FEES examinations were performed at least 6 months after completion of the HNC treatment. It is possible that patients with HNC got used to the OD symptoms and adjusted to living with the limitations as time passed [39].

The severity of the affective symptom scores in patients with HNC may be determined by many factors besides the presence of OD. For instance, social support may have a positive influence on HADS scores [40]. Factors like a psychiatric history, toxicomania, or reduced sexuality may also affect HADS scores [40, 41]. The HADS questionnaire measures the level of the affective symptom scores in general and is not specifically related to OD. However, some motor areas of the cerebral cortex seem to be important in the stress and depression connectome, and it has been suggested that anxiety might increase motor response inhibition $[42,43]$. So, it remains unclear whether the affective symptoms can be fully attributed to swallowing impairment or, conversely, whether affective symptoms might worsen OD.

The use of psychotropic drugs would presumably lead to an underestimation of the affective symptom scores. In the present study, however, the HADS scores of patients on psychotropic drugs were higher than those of patients not using this medication. Future studies should take the use of alcohol into account, as excessive alcohol consumption is often seen in patients with HNC and is associated with psychological distress [41].

In conclusion, a counter-intuitive negative association was found between the presence of aspiration and affective symptoms. Gender was also significantly associated 
with affective symptoms. The high prevalence of clinically relevant affective symptoms in all patients with HNC and OD (aspirators and non-aspirators) justifies the recommendation of a systematic screening for affective symptoms and of a subsequent collaboration between the psychosocial team and the multidisciplinary dysphagia team.

\section{Limitations}

This study has some limitations. Whereas some statistically significant results were found, the sample size was too small to allow detailed group stratification, which would be needed to detect all relevant associations. The most critical marker of OD is aspiration $[3,4]$. For this study, a previously published modified aspiration scale was used making no distinction between silent and nonsilent aspiration [26, 27]. Maybe this specification in aspiration would have been helpful in further optimizing the interpretation of the results. However, this decision to use a modified scale in the present study was based on a recent psychometric review on visuoperceptual measures in FEES and videofluoroscopy including, among others, the Penetration Aspirations Scale showing that their psychometric status was either poor or lacking data on validity, reliability, and responsiveness [44]. Finally, future research could investigate the direction of the relationship between affective symptoms and different grades of OD in patients with $\mathrm{HNC}$ in a longitudinal research design using a larger sample size. Because of the explorative nature of the study Bonferroni correction was not applied.

\section{Conclusions}

Clinically relevant affective symptoms were present on the HADS in more than half of all patients with HNC and OD (aspirators and dysphagic non-aspirators). Surpris- ingly, a significant negative association was found between the presence of aspiration and these affective symptoms. Gender was also significantly associated with affective symptoms. These results suggest that there is a need for further investigation into the impact of psychological distress on patients with $\mathrm{HNC}$ and OD.

\section{Statement of Ethics}

The study protocol was approved as non-WMO (Wet Medisch-Wetenschappelijk Onderzoek) research by the medical Ethics Committee of the Maastricht University Medical Center in compliance with the Medical Research Involving Human Subjects Act (WMO) (protocol reference number METC 2018-0855) [19]. Informed consent for using pseudonymized data was obtained from all patients and reported in the electronic patient file according to the non-WMO Act [19].

\section{Disclosure Statement}

The authors have no conflicts of interest to declare.

\section{Funding Sources}

There was no funding for this study.

\section{Author Contributions}

I.K.: substantial contributions to the conception of the study, drafting and revising, final approval, and agreement to be accountable for all aspects of the work. S.R.S.: data analysis, revision, final approval, and agreement to be accountable for all aspects of the work. W.P. and L.W.J.B.: substantial contribution to the study design, revision, final approval, and agreement to be accountable for all aspects of the work. B.K.: interpretation, revision, final approval, and agreement to be accountable for all aspects of the work. B.W.: statistics, revision, final approval, and agreement to be accountable for all aspects of the work.

\section{References}

1 Pauloski BR. Rehabilitation of dysphagia following head and neck cancer. Phys Med Rehabil Clin N Am. 2008 Nov;19(4):889-928.

2 Schindler A, Denaro N, Russi EG, Pizzorni N, Bossi P, Merlotti A, et al. Dysphagia in head and neck cancer patients treated with radiotherapy and systemic therapies: literature review and consensus. Crit Rev Oncol Hematol. 2015 Nov;96(2):372-84.

3 Ekberg O, Hamdy S, Woisard V, WuttgeHannig A, Ortega P. Social and psychological burden of dysphagia: its impact on diag- nosis and treatment. Dysphagia. 2002;17(2): $139-46$.

4 Leder SB, Espinosa JF. Aspiration risk after acute stroke: comparison of clinical examination and fiberoptic endoscopic evaluation of swallowing. Dysphagia. 2002;17(3):214-8.

5 Heijnen BJ, Speyer R, Baijens LW, Bogaardt HC. Neuromuscular electrical stimulation versus traditional therapy in patients with Parkinson's disease and oropharyngeal dysphagia: effects on quality of life. Dysphagia. 2012 Sep;27(3):336-45.
6 Speyer R, Heijnen BJ, Baijens LW, Vrijenhoef FH, Otters EF, Roodenburg N, et al. Quality of life in oncological patients with oropharyngeal dysphagia: validity and reliability of the Dutch version of the MD Anderson Dysphagia Inventory and the Deglutition Handicap Index. Dysphagia. 2011 Dec;26(4):407-14.

7 Han H, Shin G, Jun A, Park T, Ko D, Choi E, et al. The Relation Between the Presence of Aspiration or Penetration and the Clinical Indicators of Dysphagia in Poststroke Survivors. Ann Rehabil Med. 2016 Feb;40(1):88-94. 
8 Murphy BA, Ridner S, Wells N, Dietrich M. Quality of life research in head and neck cancer: a review of the current state of the science. Crit Rev Oncol Hematol. 2007 Jun;62(3):25167.

9 D’Souza V, Blouin E, Zeitouni A, Muller K, Allison PJ. An investigation of the effect of tailored information on symptoms of anxiety and depression in Head and Neck cancer patients. Oral Oncol. 2013 May;49(5):431-7.

10 Mitchell AJ, Meader N, Symonds P. Diagnostic validity of the Hospital Anxiety and Depression Scale (HADS) in cancer and palliative settings: a meta-analysis. J Affect Disord. 2010 Nov; $126(3): 335-48$.

11 Thalén-Lindström A, Glimelius B, Johansson B. Development of anxiety, depression and health-related quality of life in oncology patients without initial symptoms according to the Hospital Anxiety and Depression Scale - a comparative study. Acta Oncol. 2017 Aug; 56(8):1094-102.

12 Norton S, Cosco T, Doyle F, Done J, Sacker A. The Hospital Anxiety and Depression Scale: a meta confirmatory factor analysis. J Psychosom Res. 2013 Jan;74(1):74-81.

13 Verdonschot RJ, Baijens LW, Vanbelle S, van de Kolk I, Kremer B, Leue C. Affective symptoms in patients with oropharyngeal dysphagia: A systematic review. J Psychosom Res. 2017 Jun;97:102-10.

14 Campbell BH, Spinelli K, Marbella AM, Myers KB, Kuhn JC, Layde PM. Aspiration, weight loss, and quality of life in head and neck cancer survivors. Arch Otolaryngol Head Neck Surg. 2004 Sep;130(9):1100-3.

15 Nguyen NP, Frank C, Moltz CC, Vos P, Smith HJ, Karlsson U, et al. Impact of dysphagia on quality of life after treatment of head-andneck cancer. Int J Radiat Oncol Biol Phys. 2005 Mar;61(3):772-8.

16 Maclean J, Cotton S, Perry A. Dysphagia following a total laryngectomy: the effect on quality of life, functioning, and psychological well-being. Dysphagia. 2009 Sep;24(3):31421.

17 Pangman VC, Sloan J, Guse L. An examination of psychometric properties of the minimental state examination and the standardized mini-mental state examination: implications for clinical practice. Appl Nurs Res. 2000 Nov;13(4):209-13.

18 Edge SB, Compton CC. The American Joint Committee on Cancer: the 7th edition of the AJCC cancer staging manual and the future of TNM. Ann Surg Oncol. 2010 Jun;17(6):14714.
19 Centrale Commissie Mensgebonden Onderzoek [Internet]. Niet-WMO onderzoek [cited 2020 February 19]. Available from: https:// www.ccmo.nl/onderzoekers/soorten-onderzoek/niet-wmo-onderzoek.

20 Crary MA, Mann GD, Groher ME. Initial psychometric assessment of a functional oral intake scale for dysphagia in stroke patients. Arch Phys Med Rehabil. 2005 Aug;86(8): 1516-20.

21 Zigmond AS, Snaith RP. The hospital anxiety and depression scale. Acta Psychiatr Scand. 1983 Jun;67(6):361-70.

22 Bjelland I, Dahl AA, Haug TT, Neckelmann D. The validity of the Hospital Anxiety and Depression Scale. An updated literature review. J Psychosom Res. 2002 Feb;52(2):69-77.

23 ZUYD Meetinstrumentenzorg [Internet]. Hospital Anxiety and Depression Scale [cited 2019 May 5]. Available from: http://www. meetinstrumentenzorg.nl/.

24 International Dysphagia Diet Standardisation Initiative [Internet]. Complete IDDSI framework detailed definitions [cited 2019 March 26]. Available from: https://iddsi.org/ Documents/IDDSIFramework-CompleteFramework.pdf.

25 Sellick SM, Edwardson AD. Screening new cancer patients for psychological distress using the hospital anxiety and depression scale. Psychooncology. 2007 Jun;16(6):534-42.

26 Pilz W, Baijens LW, Passos VL, Verdonschot $\mathrm{R}$, Wesseling F, Roodenburg N, et al. Swallowing assessment in myotonic dystrophy type 1 using fiberoptic endoscopic evaluation of swallowing (FEES). Neuromuscul Disord. 2014 Dec;24(12):1054-62.

27 Baijens LW, Speyer R, Pilz W, Roodenburg N. FEES protocol derived estimates of sensitivity: aspiration in dysphagic patients. Dysphagia. 2014 Oct;29(5):583-90.

28 Rosenbek JC, Robbins JA, Roecker EB, Coyle JL, Wood JL. A penetration-aspiration scale. Dysphagia. 1996;11(2):93-8.

29 Pilz W, Vanbelle S, Kremer B, van Hooren $\mathrm{MR}$, van Becelaere $\mathrm{T}$, Roodenburg N, et al. Observers' Agreement on Measurements in Fiberoptic Endoscopic Evaluation of Swallowing. Dysphagia. 2016 Apr;31(2):180-7.

30 Landis JR, Koch GG. The measurement of observer agreement for categorical data. Biometrics. 1977 Mar;33(1):159-74.

31 Nikbakhsh N, Moudi S, Abbasian S, Khafri S. Prevalence of depression and anxiety among cancer patients. Caspian J Intern Med. 2014; 5(3):167-70.

32 Lewis S, Salins N, Kadam A, Rao R. Distress screening using distress thermometer in head and neck cancer patients undergoing radiotherapy and evaluation of causal factors predicting occurrence of distress. Indian J Palliat Care. 2013 May;19(2):88-92.
33 Lemay KR, Tulloch HE, Pipe AL, Reed JL. Establishing the Minimal Clinically Important Difference for the Hospital Anxiety and Depression Scale in Patients With Cardiovascular Disease. J Cardiopulm Rehabil Prev. 2019 Nov;39(6):E6-11.

34 Jung SJ, Kim DY, Joo SY. Rick factors associated with aspiration in patients with head and neck cancer. Ann Rehabil Med. 2011 Dec; 35(6):781-90.

35 Langerman A, Maccracken E, Kasza K, Haraf DJ, Vokes EE, Stenson KM. Aspiration in chemoradiated patients with head and neck cancer. Arch Otolaryngol Head Neck Surg. 2007 Dec;133(12):1289-95.

36 Rogus-Pulia NM, Pierce MC, Mittal BB, Zecker SG, Logemann JA. Changes in swallowing physiology and patient perception of swallowing function following chemoradiation for head and neck cancer. Dysphagia. 2014 Apr;29(2):223-33.

37 Schindler A, Ginocchio D, Peri A, Felisati G, Ottaviani F. FEESST in the rehabilitation of dysphagia after partial laryngectomy. Ann Otol Rhinol Laryngol. 2010 Feb;119(2):71-6.

38 Martino R, Beaton D, Diamant NE. Using different perspectives to generate items for a new scale measuring medical outcomes of dysphagia (MOD). J Clin Epidemiol. 2009 May; 62(5):518-26.

39 Hammerlid E, Taft C. Health-related quality of life in long-term head and neck cancer survivors: a comparison with general population norms. Br J Cancer. 2001 Jan;84(2):149-56.

40 Suzuki M, Deno M, Myers M, Asakage T, Takahashi K, Saito K, et al. Anxiety and depression in patients after surgery for head and neck cancer in Japan. Palliat Support Care. 2016 Jun;14(3):269-77.

41 Kugaya A, Akechi T, Okuyama T, Nakano T, Mikami I, Okamura H, et al. Prevalence, predictive factors, and screening for psychologic distress in patients with newly diagnosed head and neck cancer. Cancer. 2000 Jun; 88(12):2817-23.

42 Dum RP, Levinthal DJ, Strick PL. Motor, cognitive, and affective areas of the cerebral cortex influence the adrenal medulla. Proc Natl Acad Sci USA. 2016 Aug;113(35):9922-7.

43 Grillon C, Robinson OJ, O'Connell K, Davis A, Alvarez G, Pine DS, et al. Clinical anxiety promotes excessive response inhibition. Psychol Med. 2017 Feb;47(3):484-94.

44 Swan K, Cordier R, Brown T, Speyer R. Psychometric Properties of Visuoperceptual Measures of Videofluoroscopic and FibreEndoscopic Evaluations of Swallowing: A Systematic Review. Dysphagia. 2019 Feb; $34(1): 2-33$.
Patients with Head-and-Neck Cancer: Dysphagia and Affective Symptoms
Folia Phoniatr Logop 2021;73:308-315 DOI: $10.1159 / 000508367$ 